\title{
Guest Editorial: Special Issue on Discrete Geometry for Computer Imagery
}

\author{
Michel Couprie $^{1}$. Jean Cousty ${ }^{1} \cdot$ Yukiko Kenmochi $^{1} \cdot$ David Coeurjolly $^{2}$
}

Published online: 16 June 2020

(c) Springer Science+Business Media, LLC, part of Springer Nature 2020

This special issue is focused on recent developments in the fields of Discrete Geometry for Computer Imagery, which is also called Digital Geometry. Digital Geometry is indeed a subfield of discrete geometry in the sense that it focuses on geometrical objects defined on lattices and grids, in addition to the unavoidable fact that treated data are discrete. Digital Geometry aims at dealing with geometrical and topological definitions of digitized objects or digitized images and providing both theoretical and computational frameworks for image computing. In particular, it is intended to develop efficient algorithms for geometry and topology processing of digital objects. Naturally, Digital Geometry plays an essential role in various fields related to digital images, such as image analysis, computer graphics, pattern recognition, shape modeling and computer vision.

Although this domain emerged during the second half of the twentieth century with the birth of computer graphics and digital image processing, many links have been demonstrated between methods and results from the Digital Geometry domain and fundamental theorems in mathematics (arithmetic, geometry, topology, etc.), discrete mathematics (word theory, combinatorics, graph theory, etc.) or computer science (algorithmic, computational geometry, image processing, discrete tomography, computer graphics, etc.).

The center of gravity of the international Digital Geometry community lies in Europe, but there are also research groups from Australia, Brazil, India, Japan and the USA contributing to the community. In association with the International Association for Pattern Recognition (IAPR), the community collaborates within the "Discrete Geometry and Mathematical Morphology" Technical Committee, TC18. The TC18 website (http://tc18.org) also provides interested readers with a platform, which gathers useful resources such as datasets, source codes and bibliographies.

The special issue comprises eight articles covering a wide range of topics in the Digital Geometry domain, some of which are also in overlapping areas, such as Mathematical Morphology and Computational Topology. The presented articles use or develop discrete geometric and topological models, including graph and simplicial complex models, which are aimed at geometric shape digitization, distance transform computation, image segmentation, discrete optimization, digital shape analysis and geometric object recognition.

The guest editors would like to acknowledge the reviewers' invaluable contribution to this special issue and would like to thank them for their dedication.

Publisher's Note Springer Nature remains neutral with regard to jurisdictional claims in published maps and institutional affiliations.

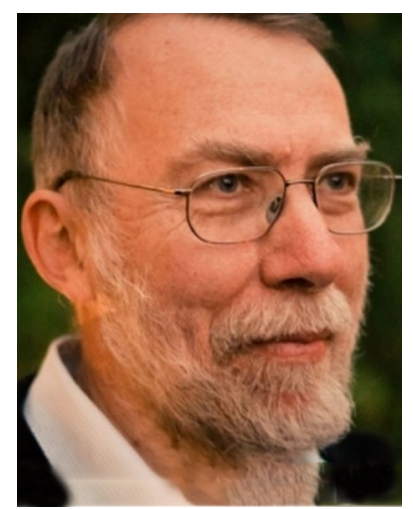

Michel Couprie received his Ingénieur's degree from the École Supérieure d'Ingénieurs en Électrotechnique et Électronique (Paris, France) in 1985, the Ph.D. degree from the Université Pierre et Marie Curie (Paris, France) in 1988, and the Habilitation à Diriger des Recherches in 2004 from the Universitéde Marne-la-Vallée (France). Since 1988 he has been working with the computer science department

2 Université de Lyon, CNRS, LIRIS, Lyon, France 
of ESIEE. He is also a member of the Laboratoire d'Informatique Gaspard-Monge, Université Paris-Est. He has authored/co-authored over 100 papers in archival journals and conference proceedings as well as book contributions. He supervised or co-supervised nine Ph.D. students who successfully defended their thesis. He is associate editor for the Pattern Recognition Letters journal. His current research interests include image analysis and discrete mathematics.

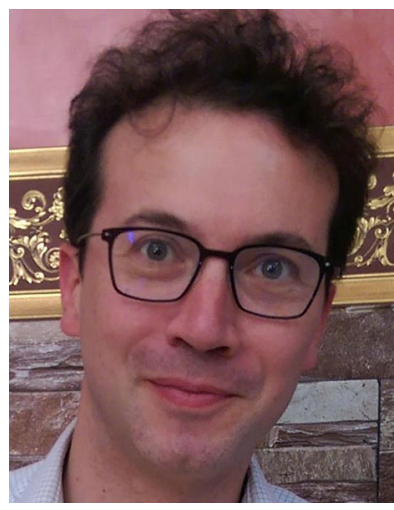

Jean Cousty received his Ingénieur's degree from the Ecole Supérieure d'Ingénieurs en Electrotechnique et Electronique (ESIEE Paris, France) in 2004, the Ph.D. degree from the Université de Marne-la-Vallée (France) in 2007 and the Habilitation á Diriger des Recherches from Université Paris-Est in 2018. After a one-year post-doctoral period in the ASCLEPIOS research team at INRIA (SophiaAntipolis, France), he is now teaching and doing research with the Informatics Department, ESIEE Paris, and with the Laboratoire d'Informatique Gaspard-Monge, Université Gustave Eiffel. His current research interests include image analysis, algorithms and discrete mathematics.

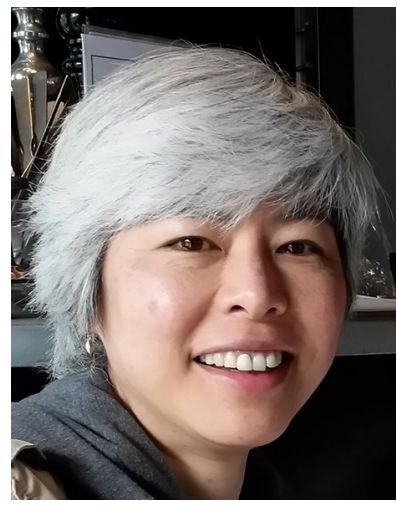

Yukiko Kenmochi is a CNRS Research Associate at Laboratoire d'Informatique GaspardMonge, Université Gustave Eiffel, where she has been since 2004. From 2003 to 2004 , she worked at Okayama University, Japan, as a Lecturer. During 1998-2003 she was a Research Associate at Japan Advanced Institute of Science and Technology. She received B.Eng., M.Eng., and D.Eng. degrees in information and computer sciences from Chiba University, Japan, in 1993, 1995, and 1998, respectively. She received her degree of Habilitation à Diriger des
Recherches from Université Paris-Est in 2018. Her research interests include discrete geometry for computer imagery.

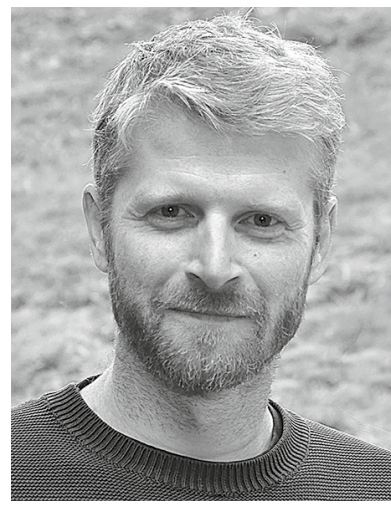

David Coeurjolly graduated from the Ecole Normale Superieure and the Universite Claude Bernard of Lyon, France, in 2000 and received the Ph.D. Degree in digital geometry in 2002 from the Université Lumiére Lyon 2. In 2003, he obtained a permanent research position (Chargé de Recherche CNRS) with the LIRIS/CNRS UMR 5205 Lyon. In 2011, he got a Senior tenured researcher position (Directeur de Recherche CNRS) at the same institute. He led the m2DisCo research team in LIRIS from

October 2011 to January 2015. From January 2017, he is director of the Fédération Informatique de Lyon (CNRS). From 2006 to 2008, he chaired the Technical Committee 18 "Discrete Geometry" of IAPR (International Association for Pattern Recognition). In 2008, he chaired and organized the International conference on Discrete Geometry for Computer Imagery (DGCI 2008) in Lyon (President of DGCI Steering Committee since 2013). In 2009, he received a Bronze Medal from CNRS that recognized his activities in Digital Geometry. His present research interests include digital and computational geometry, geometry processing and computer graphics. 